Journal of Animal and Veterinary Advances 11 (19): 3613-3617, 2012

ISSN: $1680-5593$

(C) Medwell Journals, 2012

\title{
Genetically Modified Crops and Human Health
}

\author{
Iraz Haspolat Kaya \\ Institute of Biotechnology, Ankara University, 06100 Tandogan, Ankara, Turkey
}

\begin{abstract}
Since, genetically modified (transgenic) crops have genes which do not belong to their own species, potential risks of these crops on health have brought up in recent years. In general, majority of genes transferred to plants via biotechnological methods are bacteria and virus originated which forms the basis of several problems. Since, transgenic crops include new transformed gene products and seconder metabolites, they have a potential risk on human health. The most important health risks of genetically modified crops are claimed to be allergy, toxicity and cancer. Public health regulatory systems need to be put in place in every country to identify and monitor potential adverse human health effects of transgenic crops.
\end{abstract}

Key words: Genetically modified crop, human health, adverse, cancer, transgenic

\section{INTRODUCTION}

It is well known that nutrition is associated with a lot of health conditions. Limited nutritional sources of world lead the use of alternative methods in agriculture. One of the alternative methods is the production of Genetically Modified (GM) crops. Genetically modified crops promise to solve the world's hunger and to protect the environment by increasing crop production and decreasing use of chemicals such as pesticides and herbicides (Haspolat, 2007). The sowing area of genetically modified crops which were firstly started to be cultivated as 2.8 million ha in 1996, reached to 148 million ha ( $4.63 \%$ of total world cultivated area) in 2010. After the establishment of Turkish Biosafety Law in 2010, the import of GM crops were regulated in Turkey. By the regulation of GM crops in Turkey, health issues of GM crops became a popular subject in public opinion.

\section{POSSIBLE ALLERGIC EFFECTS OF GM CROPS}

Mostly, allergic reactions that are seen in adults and children can be attributed to eight food groups. These are eggs, fish, shellfish, milk, peanuts, soybeans, tree nuts and wheat. Allergens mostly found in proteins, however among all proteins a few are allergenic and few people affected from them (Lehrer, 1999). In some surveys, it is seen that $30 \%$ people interviewed said to have at least once food allergy. However, when they are tested, this percentage decreased to $2 \%$ in adults, $7 \%$ in children. Although, there are few data that support this notion, there is a general feeling about the increase of food allergy by the public (D'Agnolo, 2005).

According to Pustzai et al. (2003) genetically modified crops are being grown and used by many people although they can carry health risks. One of these risks is possible allergic effects. One or more genes are incorporated into the crop's genome using a vector containing several other genes including as a minimum, viral promoters, transcription terminators, antibiotic resistance marker genes and reporter genes when food-crops are genetically modified. Data on the safety of these are scarce even though they can affect the safety of the GM crop. For example DNA does not always fully break down in the alimentary tract. Gut bacteria can take up genes and GM plasmids and this opens up the possibility of the spread of antibiotic resistance. Insertion of genes into the genome can also result in unintended effects which need to be eliminated by selection since some of the ways the inserted genes express themselves in the host or the way they affect the functioning of the crop's own genes are unpredictable. This may lead to the development of unknown toxic/allergenic components which researchers cannot analyze for and seriously limiting the selection criteria.

The assessment of the allergenicity of proteins from unknown allergen sources continues to be a challenge to the food industry (Lehrer and Reese, 1998). Almost all evidence suggests there is no concern about allergenic potential for proteins introduced into foods from sources with no history of allergenicity have no amino acid sequence similarities to known food allergens that are rapidly digested and that are expressed at low levels relative to the expression of major allergens. The recommended approach by amino acid sequence comparison and enzymatic digestion resistance is based on current technology available. At refining this technology, future efforts must be directed. This can be achieved through; continued allergen identification and amino acid sequence characterization to increase the 
number of allergenic sequences in the data bank; identification of the amino acid sequence properties that define allergenic epitopes to develop more exact sequence screening criteria and development of an animal model that can recognize food allergens in a manner similar to that which occurs in human disease. In spite of the fact that the technology thus far used to assess the allergenicity of GM foods can be improved, it still serves us very well in identifying potentially allergenic products that may be developed. Thus, it is possible to identify potential risks for allergenicity and minimize their effect on exposed populations. To summarize, the risk-to-benefit ratio of these new technologies must be considered. A number of serological assays are being used to reduce the risk as stated above. The benefits derived from GM crops must be considered against these risks which may vary from country to country. Allergy is a high priority among the middle and upper classes of industrial countries where any added risk in an already well-fed population may be a concern. However, in countries with emerging economies, where allergy is a lower priority than nutrition, the increased productivity benefits of GM crops may far outweigh any potential risk of allergic reactions (Lehrer, 1999).

People who eat unlabeled GM foodstuffs are mostly face allergies and anaphylaxis. It is easy to establish whether the GM food is allergenic using in vitro tests when the gene is from a crop of known allergenicity such as RAST or immune blotting with sera from individuals sensitized to the original crop. This was demonstrated in GM soybeans expressing the Brazil nut $2 \mathrm{~S}$ protein or in GM potatoes expressing code protein genes. It is also easy to assess whether genetic engineering affected the potency of endogenous allergens. Some farm workers exposed to Bacillus thuringiensis pesticide were shown to have developed skin sensitization and $\operatorname{IgE}$ antibodies to the Bt spore extract. With their sera it may now therefore be possible to test for the allergenic potential of GM crops expressing Bt toxin. It is all the more important because Bt toxin CrylAc has recently been shown to be a potent oral/nasal antigen and adjuvant.

However, assessment of the allergenicity of a GM food crop is difficult when the gene is transferred from a source not eaten before or with unknown allergenicity or on gene insertion a new allergen or adjuvant is developed or the expression of a minor allergen is increased. Unfortunately, while there are good animal models for nutritional or toxicological testing such models do not exist for allergenicity testing.

Only indirect and rather scientifically unsound methods such as finding short sequence homologies (at least 8 contiguous amino acids) to about 200 known allergens are used for the assessment of allergenicity. The decision-tree type of indirect approach based on factors such as size and stability of the transgenically expressed protein is more unsound, particularly as its stability to gut proteolysis is assessed by an in vitro (simulated) testing instead of in vivo (human/animal) testing and this is fundamentally wrong. The concept that most allergens are abundant proteins is also misleading because for example Gad c1, the major allergen in codfish is not a predominant protein. When the gene responsible for the allergenicity is known such as the gene of the alpha-amylase/trypsin inhibitors/allergens in rice, cloning and sequencing opens the way for reducing their level by antisense RNA strategy. In the absence of reliable methods for allergenicity testing, it is currently impossible to definitely establish whether a new GM crop is allergenic or not before its release into the human-animal food-feed chain (Pusztai, 2001).

Among all food allergies, nuts are among the most important allergy sources and allergies to the Brazil nut are searched till now. Concern has been expressed about the introduction of allergenic proteins into food plants by genetic engineering. While studying about Brazil nuts it is intended to determine whether the $2 \mathrm{~S}$ albumin from the Brazil nut as expressed in transgenic soybeans was able to bind $\operatorname{IgE}$ from people who are allergic to Brazil nuts. The $2 \mathrm{~S}$ albumin from the Brazil nut is most likely a major allergen. Major allergens are proteins that bind substantially to $\operatorname{IgE}$ from $>50 \%$ of the patients with that specific allergy. In a study, serum from eight of nine subjects allergic to Brazil nuts recognized the $2 \mathrm{~S}$ albumin as a major IgE-binding protein of the Brazil nut and this protein was by far the strongest $\operatorname{IgE}$-binding protein in seven of the eight subjects. IgE from these seven subjects also bound to the $2 \mathrm{~S}$ albumin in transgenic soybeans which carry the Brazil nut 2S albumin gene. Moreover, skin-prick tests with extracts of transgenic soybean were positive in the three subjects allergic to Brazil nuts who were tested whereas tests with nontransgenic-soybean extract were negative. Although, oral challenges would be necessary to confirm the allergenicity of the $2 \mathrm{~S}$ albumin, they would pose a risk to this group which consists of people that experience life-threatening symptoms on inadvertent consumption of Brazil nuts. It is crucial to assess the allergenicity of proteins in transgenic foods if those proteins have been derived from sources that are commonly allergenic. The use of currently available animal models alone to predict allergenicity in humans does not produce accurate results (Nordlee et al., 1996). 


\section{POSSIBLE TOXIC EFFECTS OF GM CROPS}

The World Health Organization (WHO) explains that Genetically Modified Organisms (GMOs) are the organisms with the genetic substance that is changed unnaturally. Chosen individual genes are carried from one organism into another and between species that are not associated with each other by the technology that is used. Genetically modified plants are created by these methods. Then, GM food crops are grown by these genetically modified plants.

Because that different GMOs have different genes added in different ways, the WHO specifies that individual foods and their safety must be evaluated in detail. The safety evaluation of GM foods should examine toxicity, allergenicity, specific elements that may have nutritional or toxic characteristics, steadiness of the added gene, nutritional results related with genetic alteration and any unintended results that may derive from the gene insertion (Domingo, 2007).

Potatoes: For the group of mice that consumed delta-endotoxin-treated potatoes some little changes were seen in the structural shape of their ileum. The length of this study was 2 weeks. However, when this study applied for a group of rats, the length of the study changed. For 10 days of this study, reproduction of the gastric mucosa and effects on the small intestine and caecum were observed. When this study lasted for 4 weeks, there were no pathologic signs and histopathological irregularities in liver and kidney. When it lasted for 5 weeks, the number of bacteria which were phagocytized by monocytes, the number of neutrophils that develop ROS and the number of oxygen-dependant bactericidal operation of neutrophils was expanded. For the length of the study which was 10 weeks before mating, there wasn't any contrary effect on the multigenerational reproductive-developmental skill.

Maize/corn: To decide the effect of transgenic event 176-derived corn (Event 176 indicates a gene that enables the plants to produce an insecticidal protein, $\mathrm{Cryl} \mathrm{Ab}$ ) on chickens a study with the length of 38 days was made. As a result, there weren't any important differences and effects in body weight. When this study was applied on pigs for 91 days, side effects were not examined but the operation of toxicological tests was not also specified by these studies. When this study lasted for 13 weeks and applied on rats and mice, immunotoxicity was not observed and other particular toxicity tests were not involved. Also, when it was applied for rats for 13 weeks, there weren't any contrary results on health, body weight, food consumption, clinical pathology parameters, organ weights as well as total and microscopic appearance of tissues.

Soybeans: For this study, glyphosate-tolerant soybeans were used on rats, broiler chickens, catfish and dairy cows. The study lasted for 4 weeks for rats and cows, 6 weeks for broiler chickens and 10 weeks for catfish. However, for all of them, important effects were not observed in the concentrations of nutrients and antinutrients. Another genetically modified soybean 40-3-2 was used on rats for 5 months. As a result of this study, changes in the hepatocyte membrane task and enzymatic function were seen within physiological levels. Glyphosate-tolerant soybeans were used on rats and mice for 15 weeks and no contrary results on growth and the histopathology of immune-related organs were observed. There wasn't also any immunotoxic activity. When this study was applied on pigs during the growing period, it did not show the performance of toxicological tests. When it was applied on rats for 13 weeks again no contrary effects of this GM soybean meal was observed. Also, when it was applied on mice during maternity and lactation periods, there were not any negative results on fetal, postnatal, pubertal or adult testicular growth.

Rice: Genetically modified rice with soybean glycine was given to rats for 4 weeks and it was observed that there weren't any contrary effects on the blood count, blood composition or internal organ weighs. There weren't also any pathological symptoms or histopathological regularities in liver and kidney. Rice with anti-herbicide gene $(B A R)$ was also given to mice and rats for 30 days. Again, there were not any contrary effects on body or histopathological changes. Rica with cowpea trypsin inhibitor was also used for rats during the period from lactation to sexual maturation but no maternal toxicity, embryo toxicity and teratogenicity were observed. However, when this kind of rice was given to rats for 90 days, some changes on hematological parameters were observed. When it was given to mice for 30 days, there were not any immunotoxic results and other toxicity tests were not executed. When the transgenic rice was given to rats for 90 days, there were not any sufficient suggestions which indicate that transgenic rice had contrary effects on the rat. When the transgenic KMD1 rice was given to rats for 90 days, little changes were seen but other tests were needed.

Other GM crops: When the transgenic cucumber was given to rats for 5 weeks no contrary effects were 
detected on the growth and health condition. When the genetically modified tomatoes were given to rats for 90 days, there was not any abnormality in their body weights and food consumption. Also, when the microscopy examination was made on tissues, contrary effects were not seen. When these GM tomatoes were given to rats and mice for 30 days there were not any important differences with rats which ate non-GM tomatoes. When GM sweet pepper was given to rats and mice for 30 days, there were not any important differences with those which ate non-GM sweet peppers. When the transgenic peas were given to rats for 10 days there were not any damaging results on growth, metabolism and health. When the transgenic canola plants were given to rats for 26 days, it was observed that there were not any general health risks (Lehrer and Reese, 1998; Domingo, 2007; Bakshi, 2003).

In addition to these studies, three articles on genetically modified plants' effects have been reviewed. One of these studies is Zdunczyk (2001)'s study which indicates that evaluation of the suitability of the chemical structure of transgenic and traditional crops are not sufficient. Another study is the Bakshi (2003)'s study which is about the contrary health effects of genetically modified crops. This study also indicates that because of the newness of the technology that is used for genetic engineering of crops, scientists have an insufficient understanding of physiology, genetics and nutritional value of genetically engineered crops. Thus, it also indicates that some risks may not be identified and that the genetically modified crops may have other toxic substances. The other study is Pryme and Lembcke (2003)'s study which concludes that more scientific effort and investigation are necessary in order to confirm that foods with genetically modified substance do not cause any serious health problems in the long term.

Other study about the evaluation of health risk of GMOs is made by Monsanto Company which used a transgenic corn MON863 in order to implement this study. This study was applied on rats for 90 days and comparisons between rats fed with GMO and rats with an equivalent normal diet and individually with six recommended diets with different compounds were examined. During this study it was observed that after the consumption of MON863, there were little but dose-related important differences in growth for both males and females. There was a $3.3 \%$ decrease in weight for males and $3.7 \%$ increase for females. These measurements showed that there was hepatorenal toxicity and noticeable different sensitivities in males and females (Seralini et al., 2007). Also, triglycerides increased by $24-40 \%$ in females while urine phosphorus and sodium excretions decreased in males by $31-35 \%$ compared to seven diets that were examined. However, it was concluded that longer experiments were necessary to confirm the real nature and scope of the pathology because with these measurements it was hard to say that GM corn MON 863 was a safe and a non-toxic product.

Another research paper is about the effects of three GM corn differences on mammalian health. This study compares the blood and organ system information from the experiments with rats that were given three GM maize (NK 603, MON 810 and MON 863) which are found in food and feed. After 60 different biochemical parameters were classified per organ and measured in serum and urine after 5 and 14 weeks of feeding, the analysis showed that these three GM maize had side effects which were dependent on sex and dose. These effects were mostly on kidney and liver. Other effects were shown in the heart, adrenal glands, spleen and hematopoietic system. These results confirm that there existed hepatorenal toxicity because of the new pesticides found on each GM corn (De Vendomois et al., 2009).

Patho-physiological analysis are single for each GM food because comments related to general and similar sub chronic toxic effects for all GM foods cannot be made. In addition, all three GM maize differences have a different pesticide residue related to their specific GM event (glyphosate and AMPA in NK 603, modified Cryl Ab in MON 810, modified Cry3Bbl in MON 863). However, longer (up to 2 years) animal feeding studies should be analyzed to confirm the toxic effects of GM crops, feed and foods. In addition, this study showed that the kidneys and liver are the organs that should be focused on because of the analysis of the negative effects on the function of these organs after the study of rats which consumed GM maize for 90 days.

\section{POSSIBLE CARCINOGENIC EFFECTS OF GM CROPS}

According to Direct Cancer and Degenerative Disease Links in 1994, FDA approved Monsanto's rBGH, a genetically produced growth hormone for injection into dairy cows even though scientists warned the resulting increase of IGF-1, a potent chemical hormone, is linked to $400-500 \%$ higher risks of human breast, prostrate and colon cancer. Dr. Samuel Epstein of the University of Chicago demonstrates that it induces the malignant transformation of human breast epithelial cells. Rat studies confirmed the suspicion and showed internal organ damage with rBGH ingestion. The FDA's own experiments indicated a spleen mass increase of $46 \%$ a sign of developing leukemia. The contention was that the hormone was killed by pasteurization. 
Two Monsanto scientists, Ted Elasser and Brian McBride conducted a research and according to that research only $19 \%$ of the hormone was destroyed despite boiling milk for $30 \mathrm{~min}$ when normal pasteurization is $30 \mathrm{sec}$. Canada, the European Union, Australia and New Zealand have banned rBGR, an international health standards setting body. The UN's Codex Alimentarius refused to certify $\mathrm{rBGH}$ as safe. Furthermore and according to researcher Sharyn Martin, a number of autoimmune diseases are enhanced by foreign DNA fragments that are not fully digested in the human stomach and intestines. DNA fragments are absorbed into the bloodstream, potentially mixing with normal DNA. The genetic consequences are unpredictable and in GM soy crops unexpected gene fragments have shown up (Batalion, 2000).

\section{REFERENCES}

Bakshi, A., 2003. Potential adverse health effects of genetically modified crops. J. Toxicol. Environ. Health Part B: Crit. Rev., 6: 211-225.

Batalion, N., 2000. 50 Harmful effects of genetically modified foods. Americans for Safe Food, Oneonta, New York, USA. http:// www. scribd. com/doc/ 9467203/50-Harmful-Effects-of-Genetically-ModifiedFoods.

D'Agnolo, G., 2005. GMO: Human health risk assessment. Vet. Res. Commun., 29: 7-11.

De Vendomois, J.S., F. Roullier, D. Cellier and G.E. Seralini, 2009. A comparison of the effects of three GM corn varieties on mammalian health. Int. J. Biol. Sci., 5: $706-726$.

Domingo, J., 2007. Toxicity studies of genetically modified plants: A review of the publishes literature. Crit. Rev. Food Sci. Nutr., 47: 721-733.
Haspolat, I., 2007. The trade of genetically modified agricultural crops. Gazi Univ. J. Vocational Educ., 9: $58-75$.

Lehrer, S. and G. Reese, 1998. Food Allergens: Implictions for Biotechnology. In: Biotechnology and Safety Assessment, Thomas, J. (Ed.). 2nd Edn. Taylor and Francis Press, USA., pp: 127-150.

Lehrer, S.B., 1999. Potential Health Risks of Genetically Modified Organisms: How can Allergens be Assessed and Minimized? In: Agricultural Biotechnology and the Poor, Persley, G.J. and M.M. Lantin (Eds.). Consultative Group on International Agricultural Research, USA., pp: 149-155.

Nordlee, J.A., S.L. Taylor, J.A. Townsend, L.A. Thomas and R.K. Bush, 1996. Identification of a Brazil nut allergen in transgenic soybeans. New Eng. J. Med., 334: 688-692.

Pryme, I.F. and R. Lembcke, 2003. In vivo studies on possible health consequences of genetically modified food and feed-with particular regard to ingredients consisting of genetically modified plant materials. Nutr. Health, 17: 1-8.

Pustzai, A., S. Bardocz and S.W.B. Ewen, 2003. Genetically Modified Foods: Potential Human Health Effects. In: Food Safety Contaminants and Toxin, D'Mello, J.P.F. (Ed.). CABI Publisher, UK., pp: 347-372.

Pusztai, A., 2001. Genetically modified foods: Are they a risk to human/animal health? An Actionbioscience. Org Original Article, http:/www.actionbioscience. org/biotech/pus ztai.html.

Seralini, G.E., D. Cellier and J.S. De Vendomois, 2007. New analysis of a rat feeding study with a genetically modified maize reveals signs of hepatorenal toxicity. Arch. Environ. Contam. Toxicol., 52: 596-602.

Zdunczyk, Z., 2001. In vivo experiments on the safety evaluation of GM components of feeds and foods. J. Anim. Feed Sci., 10: 195-210. 